\title{
Double monodromy inflation: Gravitational wave factory for CMB-S4, LiteBIRD, and LISA
}

\author{
Guido D’Amico $\odot,{ }^{1,2, *}$ Nemanja Kaloper® ${ }^{3, \dagger}$ and Alexander Westphal ${ }^{4, \$}$ \\ ${ }^{1}$ Department of Mathematical, Physical and Computer Sciences, University of Parma, 43124 Parma, Italy \\ ${ }^{2}$ INFN Gruppo Collegato di Parma, 43124 Parma, Italy \\ ${ }^{3}$ QMAP, Department of Physics, University of California, Davis, California 95616, USA \\ ${ }^{4}$ Deutsches Elektronen-Synchrotron DESY, Theory Group, D-22603 Hamburg, Germany
}

(Received 24 June 2021; accepted 23 September 2021; published 21 October 2021)

\begin{abstract}
We consider a short rollercoaster cosmology based on two stages of monodromy inflation separated by a stage of matter domination, generated after the early inflaton falls out of slow roll. If the first stage is controlled by a flat potential, $V \sim \phi^{p}$ with $p<1$ and lasts $\mathcal{N} \sim 30-40 e$-folds, the scalar and tensor perturbations at the largest scales will fit the CMB perfectly, and produce relic gravity waves with $0.02 \lesssim r \lesssim 0.06$, which can be tested by LiteBIRD and CMB-S4 experiments. If in addition the first inflaton is strongly coupled to a hidden sector $U(1)$, there will be an enhanced production of vector fluctuations near the end of the first stage of inflation. These modes convert rapidly to tensors during the short epoch of matter domination, and then get pushed to superhorizon scales by the second stage of inflation, lasting another 20-30 $e$-folds. This band of gravity waves is chiral, arrives today with wavelengths in the range of $10^{8} \mathrm{~km}$, and with amplitudes greatly enhanced compared to the long wavelength CMB modes by vector sources. It is therefore accessible to LISA. Thus our model presents a rare early universe theory predicting several simultaneous signals testable by a broad range of gravity wave searches in the very near future.
\end{abstract}

DOI: 10.1103/PhysRevD.104.L081302

\section{INTRODUCTION}

Inflation [1-3] arose as a paradigm to explain the universe naturally. It has been noted well prior to its advent that the universe is incredibly unnatural at the largest scales [4]. The mechanism of inflation addresses these problems by reducing the sensitivity to the initial conditions, metaphorically taking the log of the measure of tuning: one needs a total of $\sim 60 e$-folds to shield from bad influences of initial anisotropies and inhomogeneities. As a bonus one receives a mechanism to generate structures at shorter scales, using local and causal dynamics enshrined in the effective field theory (EFT) of the inflaton sector.

Thus inflation translates the problem of cosmological naturalness to the problem of naturalness of the inflaton EFT. The construction of natural EFTs is relatively straightforward in the limit of semiclassical gravity. For example, all one needs is a single field with a flat potential

\footnotetext{
*damico.guido@gmail.com

†kaloper@physics.ucdavis.edu

*alexander.westphal@desy.de
}

Published by the American Physical Society under the terms of the Creative Commons Attribution 4.0 International license. Further distribution of this work must maintain attribution to the author(s) and the published article's title, journal citation, and DOI. Funded by SCOAP. and derivative couplings to everything else, and a model is born [5]. However with full-on quantum gravity, building UV complete inflation models is quite challenging. One general approach which was initiated in the past decade or so is monodromy inflation [5-8], where the natural EFTs of the inflaton can be protected from the perils of quantum gravity by embedding them into gauge theories spontaneously broken at some scale above the scale of inflation, but below the fundamental gravity scale.

Even so, deploying monodromy models is nontrivial, with some pressure coming from both the theory side due to backreaction induced in setups with large field variations, and the observations, because the dynamics predicts large amplitude primordial gravity waves. We stress that backreaction is not automatically detrimental, since corrections often turn beneficial by flattening inflaton potentials and prolonging inflation instead of shortening it [9-12]. The flatter potentials however tend to make the spectral index bluer. This seems to narrow the remaining theory space for these models.

In this paper we argue that such a view is far too pessimistic. Within the recently expounded framework of rollercoaster cosmology [13] (see also [14-19]), monodromy models remain completely viable, fitting the observations perfectly while relaxing the theoretical pressure from large field variations. More importantly, they are very predictive, producing primordial gravity waves within reach of both the future CMB searches such as LiteBIRD and CMB-S4, and 
the shorter scale instruments such as LISA. The reason is that the early stage of inflation can be completely interrupted, ending with a rapid reheating and then followed by another stage involving a completely different, second inflaton [13].

If the first stage lasts some $30-40 e$-folds, the flattened monodromy models with $V \sim \phi^{p}, p \lesssim 1 / 2$ [9-12] easily produce the spectra of scalar and tensor perturbations completely within the current observational limits, with $n_{s} \sim$ 0.965 and $r \lesssim 0.06$ (depending on $p$ and number of $e$-folds, we can have a slightly larger upper bound for $r$ ). The flattening of the potential reduces $r$. Since the predictions are calculated at the pivot point of $N \sim 30-40 e$-folds before the end of the first stage of inflation, $n_{s}$ is more red. Hence both $n_{s}$ and $r$ move toward the observationally favored regime. Note also that the flattening dynamics which generates very shallow potentials activates other irrelevant operators, including higher derivative corrections to EFT [9-12]. These terms yield equilateral non-Gaussianity $f_{N L}^{e q} \simeq \mathcal{O}(1)$ at $\mathrm{CMB}$ scales [12], again potentially accessible to detection. This also yields a lower bound on $r$, which makes the models quite predictive. Note further that additional stronger nonGaussianities can be produced at shorter scales, during the interruption between the two stages of inflation, when the fields turn in the field space [20] (see [21] for an extensive review). Those are however not directly observable at present.

Further, since the inflatons are axions, they generically come from dimensional reduction of higher rank forms which have anomalous couplings. As a result the UV completion of inflatons yields the usual dimension-5 operators $\propto \phi F_{\mu \nu} \tilde{F}^{\mu \nu} / f$ in four dimensions, where $\tilde{F}_{\mu \nu}=$ $\epsilon_{\mu \nu \lambda \sigma} F^{\lambda \sigma} / 2$. When the field $\phi$ depends on time, such as an inflaton in the early stage, and the time dependence is reasonably fast, such as near the end of the first stage of inflation, one of the gauge field helicities becomes tachyonic at large wavelengths [22] and the tachyonic instability leads to a rapid, nonperturbative generation of the field $F$. In turn $F$ sources chiral gravity waves $[23,24]$ with an amplitude much greater than the nonchiral gravity waves generated by the standard metric fluctuations $[13,25,26]$. The dominant chiral gravity waves become superhorizon just before the end of the first stage of inflation, and their enhanced amplitude remains frozen during the short epoch of matter domination preceding the second stage of inflation, which provides additional 20-30 $e$-folds [13]. During the last stage, the wavelength of the frozen chiral tensors stretches to the range of $10^{8} \mathrm{~km}$ which make them accessible to LISA.

Thus it would appear that the issues normally interpreted as theoretical challenges to inflation might instead be viewed as aspects of naturalness influenced by quantum gravity. Quantum gravity forces a modification of the flat space naturalness arguments; however the effect of these modifications need not be detrimental. On the contrary, monodromy inflatons with restricted field range, due to e.g., strong coupling effects, nevertheless naturally realize long inflation by using multiple inflatons working in unison. It turns out that in this sense these models are not only natural, but also more predictive than uninterrupted inflation, yielding detectable signals over a broad range of wavelengths.

\section{WHITHER DOUBLE-COASTER?}

In the rest of this work we will specialize to a simple twofield model in which inflation is realized in two stages, connected by a phase during which the original inflaton field oscillates, so that the effective equation of state of the universe is approximately $w=0$, like CDM. Such models naturally occur in monodromy constructions, involving multiple fields with little hierarchies between their masses, and flattened effective potentials at large field ranges. We will use

$V\left(\phi_{1}, \phi_{2}\right)=M_{1}^{4}\left[\left(1+\frac{\phi_{1}^{2}}{\mu_{1}^{2}}\right)^{\frac{p_{1}}{2}}-1\right]+M_{2}^{4}\left[\left(1+\frac{\phi_{2}^{2}}{\mu_{2}^{2}}\right)^{\frac{p_{2}}{2}}-1\right]$.

The scales $\mu_{1}, \mu_{2}$ normalizing the fields are both $\mathcal{O}\left(0.1 M_{\mathrm{Pl}}\right)$. We take $M_{2} \lesssim M_{1}$.

Here we assume both fields $\phi_{i}$ are axions arising from truncating $p$-form gauge potentials in string theory compactifications. This immediately gives the mass scales $\mu_{i} \sim f_{i}$ linked to the axion decay constants. These in turn are bounded by $f \sim M_{\mathrm{Pl}} /\left(M_{s} L\right)^{q} \lesssim M_{\mathrm{Pl}}$ where $L$ is the size of the compactification cycle giving rise to the relevant axion, and $M_{s}$ the string scale $[27,28]$. Hence, $f_{i}$ typically fall in the range $10^{-2} M_{\mathrm{Pl}} \lesssim f \lesssim M_{\mathrm{Pl}}$, justifying our choice of $\mu_{i}$. The scales $M_{i}$ typically arise from warping effects or dilution of energy densities with inverse powers of extra dimension volumes (see e.g., Sec. 4.1 in [29] for a summary). These are either power-law and/or exponentially sensitive to the underlying microscopic parameters of a string compactification, and the axions $\phi_{i}$ arise from two different sectors of a given model. Hence generically $M_{1} \neq M_{2}$ and thus without loss of generality $M_{2} \lesssim M_{1}$.

These choices decouple the early inflationary trajectory from the late inflationary dynamics. Therefore we can study the dynamic as a sequence of two consecutive single-field stages. We can view this as a very simple realization of Nflation [30], with a larger mass gap between the two inflatons, where one originally dominates but falls out of slow roll well before the other field does. The potential is illustrated in Fig. 1.

First, we determine the spectrum of perturbations on large scales. We are using the effective potential during the first stage

$V_{\mathrm{eff}}\left(\phi_{1}\right)=M_{1}^{4}\left[\left(1+\frac{\phi_{1}^{2}}{\mu_{1}^{2}}\right)^{\frac{p_{1}}{2}}-1\right] \simeq M_{1}^{4}\left[\left(\frac{\phi_{1}}{\mu_{1}}\right)^{p_{1}}-1\right]$,

which in the plot of Fig. 1 corresponds to fixing $\phi_{2}$ at some value and rolling down the hill toward the ridge at a $\phi_{2} \simeq$ const, thanks to $\phi_{2} \gg \mu_{2}$. Explicitly, we define the end of the first stage by $\epsilon=-\dot{H} / H^{2} \simeq 1$, which corresponds to roughly $\phi_{1}$ (end) $\simeq \mu_{1}$ for our values of $\mu_{k}$, and use the slow-roll expressions $n_{s}-1=2 \eta_{V}-6 \epsilon_{V}, r=16 \epsilon_{V}$ where 


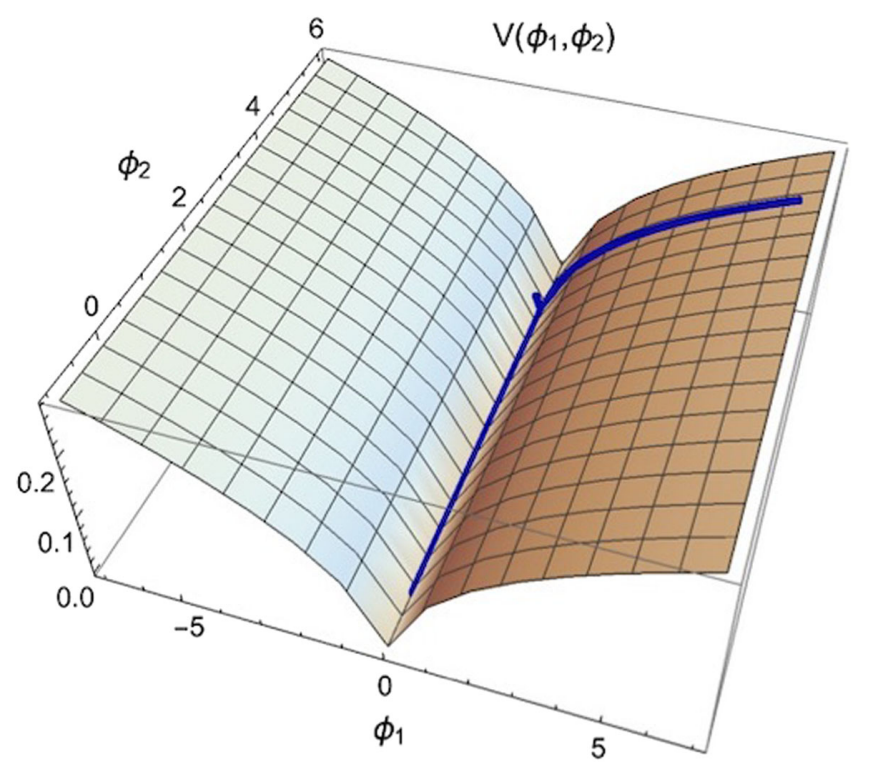

FIG. 1. Two-field potential $V\left(\phi_{1}, \phi_{2}\right)$ for the model in Eq. (1). Here $M_{1}=0.5 M_{\mathrm{Pl}}, M_{2} / M_{1}=0.1, p_{1}=2 / 5, p_{2}=1, \mu_{1}=\mu_{2}=0.5$. The blue curve depicts a typical two-stage inflationary trajectory.

$\epsilon_{V}=\partial_{\phi} V_{\text {eff }}\left(\phi_{1}\right)^{2} /\left(2 V_{\text {eff }}\left(\phi_{1}\right)^{2}\right), \eta_{V}=\partial_{\phi}^{2} V_{\text {eff }}\left(\phi_{1}\right) / V_{\text {eff }}\left(\phi_{1}\right)$, calculated at a value of $\phi_{1}$ when

$N_{e}=\int_{\phi_{1}}^{\phi_{1}(\text { end })} d \phi \frac{H}{\dot{\phi}} \simeq\left(\frac{\phi_{1}^{2}}{2 p_{1} M_{\mathrm{Pl}}^{2}}\right)\left[1-\frac{2}{2-p_{1}}\left(\frac{\mu_{1}}{\phi_{1}}\right)^{p_{1}}\right]$

$e$-folds before the end of the first inflationary stage. ${ }^{1}$ As $\mu_{1}$ decreases, $N_{e}$ increases for fixed $p_{1}$ and $\phi_{1}$. This is since as $\mu_{1}$ is scaled down the field ventures to flatter regions, and hence inflation runs longer, with $n_{s}$ shifting slightly toward less red values. With the hierarchy between inflaton scales, this suppresses non-Gaussianities during the initial stage [20].

In Fig. 2 we display our results similarly to [29], plotting predictions for different choices of parameters, at three choices of $e$-folds before the end of the first stage of inflation: $N_{e}=55,40,35$. Clearly double monodromy inflation is fully compatible with the data. An interesting prediction is that $r \gtrsim 0.02$, which is well within the reach of near future B-modes searches (see, e.g., [31]).

Restricting $\Delta \phi$ 's to be at most a few $M_{\mathrm{Pl}}$, as per the current lore about large field variations (e.g., [33-35]), we see that the individual stages are pretty short. For example, for $x=\phi_{\max } / M_{\mathrm{Pl}} \sim 2-3$, with our values of $\mu$, Eq. (3) yields for, e.g., $p \sim 0.1, N_{\max } \sim 20-45$, and $\Delta \phi$ during inflation is at most 2-3 $M_{\mathrm{Pl}}$. With slightly larger $p$ the stages of inflation are shorter. Assuming a uniform distribution of field values, it is quite generic to obtain a multistage model which, with initial value of $\phi$ 's saturating at $\phi_{\max }$ will yield a longer earlier stage and a shorter later stage, realizing a double-coaster of $\sim 40$ and $\sim 20$ stages

\footnotetext{
${ }^{1}$ Other terms may correct (3) near the end of the first stage of inflation; here we neglect such corrections.
}

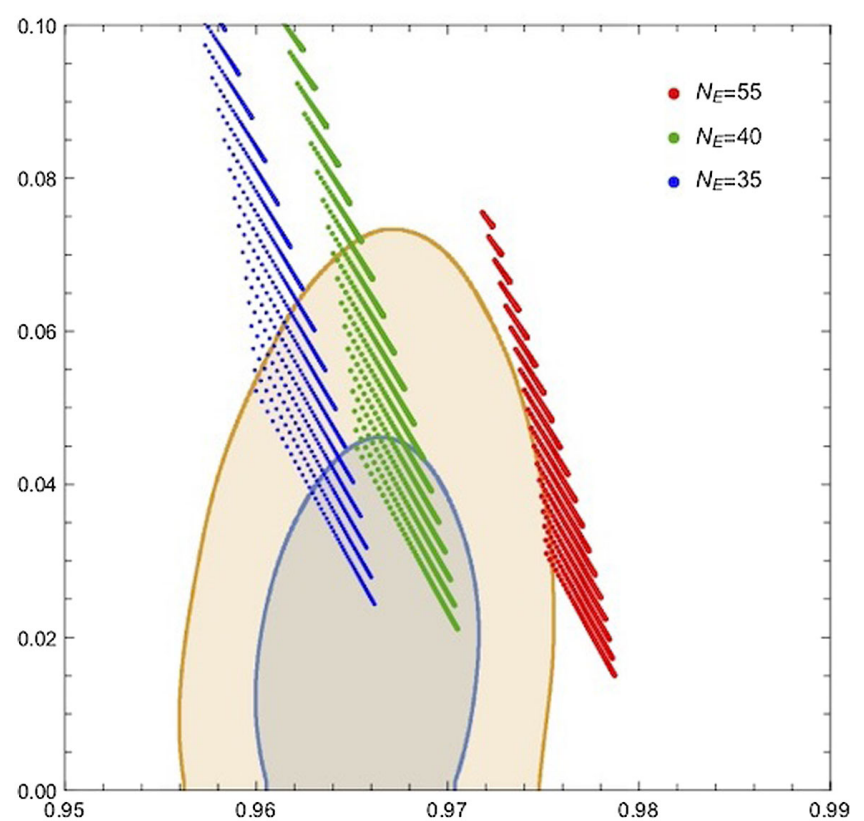

FIG. 2. Tensor-to-scalar ratio versus spectral index for double monodromy, compared to the data of [32]. We show predictions for the parameter ranges $0.01 \leq \mu_{1} \leq 1,0.1 \leq p_{1} \leq 1$. The lines in the direction of decreasing $r$ and increasing $n_{s}$ correspond to varying $\mu_{1}$ at fixed $p_{1}$; the ones at approximately constant $n_{s}$ correspond to varying $p_{1}$ at fixed $\mu_{1}$. As $\mu_{1}$ decreases, $N_{e}$ is larger since the potential is flatter at a fixed $\phi$ and so $n_{s}$ increases.

separated by a brief epoch of matter domination supported by the decay of the early inflaton. The small price to pay is to arrange for the right combination of values of $p_{k}, \mu_{k}, M_{k}$.

Note that in the first stage, the primordial spectrum of tensors remains at $r \lesssim 0.06$ at all scales at which the metric fluctuates, by scale invariance. The transition between different stages of inflation would not suddenly make this contribution jump up at shorter scales as noted by $[13,25,26]$. This opens the door for the nonperturbative generation of chiral tensors, using vector tachyon instability [23,24].

\section{FÜR LISA}

Axion inflatons generically couple to $U(1)$ gauge fields via the standard dimension-5 operators $\propto \phi_{1} F_{\mu \nu} \tilde{F}^{\mu \nu} / 2$. This can be seen in a particularly simple way in flux monodromy models $[5,8,36]$. Imagine that the axion sector arises from a dimensional reduction of, say, a 4-form field strength in $11 D$ Sugra, which has Chern-Simons self-couplings. Ignoring the volume moduli, and imagining a toroidal compactification for simplicity, we see that the $11 D$ Lagrangian upon dimensional reduction and truncation to zero modes yields

$$
\begin{aligned}
& -F_{a b c d}^{2}+\epsilon_{a_{1} \ldots a_{11}} A^{a_{1} \cdots} F^{a_{4} \cdots} F^{a_{8} \ldots a_{11}} \\
& \quad \ni-F_{\mu \nu \lambda \sigma}^{2}-\left(\partial \phi_{1}\right)^{2}-\mu \phi_{1} \epsilon_{\mu \nu \lambda \sigma} F^{\mu \nu \lambda \sigma} \\
& \quad-\sum_{k} F_{\mu \nu(k)}^{2}-\frac{\phi_{1}}{f_{\phi}} \sum_{k, l} \epsilon_{\mu \nu \lambda \sigma} F^{\mu \nu}{ }_{(k)} F^{\lambda \sigma}{ }_{(l)},
\end{aligned}
$$


where the first line involves the 4-form-axion sector, and the second the mixing of the axion with the $U(1)$ coming from the reduction of $F_{a b c d}$. Here, $A_{a b c}$ is the 3-form potential, $F=d A$. The dimensional normalizations come from different scaling dimensions of various spins, and emerge after the size of internal cycles are accounted for [37]. After rotating modes in the $U(1)$ isospace and canonically normalizing the $4 D$ fields, we see that $\phi_{1}$ will couple to at least one $4 D U(1)$ vector field. For simplicity, we take only one coupling to be nonzero, and model it with the canonically normalized $4 D$ dimension-5 operator

$$
\mathcal{L}_{\text {int }}=-\sqrt{-g} \frac{\phi_{1}}{4 f_{\phi}} F_{\mu \nu} \tilde{F}^{\mu \nu},
$$

where $f_{\phi}$ is sub-Planckian, and generically of the order of GUT scale (see, e.g., [27,28]). A rolling axion triggers the tachyonic instability of one circular polarization of the gauge field [22], whose exponential production both backreacts on the inflaton and produces scalar and tensor perturbations $[23,24]$. The dynamics is governed by [38]

$$
\begin{aligned}
& \ddot{\phi}_{1}+3 H \dot{\phi}_{1}+\partial_{\phi_{1}} V\left(\phi_{1}\right)-\frac{1}{f_{\phi}}\langle\vec{E} \cdot \vec{B}\rangle=0, \\
& 3 H^{2}=\frac{\dot{\phi}_{1}^{2}}{2}+V\left(\phi_{1}\right)+\frac{1}{2} \rho_{E B}, \\
& A_{ \pm}^{\prime \prime}(\tau, \vec{k})+\left[k^{2} \pm 2 \lambda \xi k a H\right] A_{ \pm}(\tau, \vec{k})=0,
\end{aligned}
$$

where the dot denotes a derivative with respect to $t$, and the prime is a derivative with respect to conformal time $\tau$. The $U(1)$ "electric" and "magnetic" fields are defined as usual in the Coulomb gauge, $\vec{E}=-\frac{1}{a^{2}} \frac{\mathrm{d} \vec{A}}{\mathrm{~d} \tau}, \vec{B}=\frac{1}{a^{2}} \vec{\nabla} \times \vec{A}$, and $\rho_{E B}=$ $\left(\vec{E}^{2}+\vec{B}^{2}\right) / 2$ is the standard U(1) energy density. We picked the usual circular helicity basis for $\vec{A}$ in (6). Further $\lambda$ is the sign of $\dot{\phi}_{1}$ and $\xi=\frac{1}{2 f_{\phi}} \frac{d \phi_{1}}{d N_{e}}$, where $N_{e}$ is the $e$-fold clock reading, for notational convenience.

The key ingredient here is the vector field equation. When $\dot{\phi}_{1} \neq 0$, and for $2 \xi>k /(a H)$, the gauge field helicity $-\lambda$ behaves like a tachyon, and is exponentially produced by the evolution of $\phi_{1}$ [39]. This is the case during inflation. Approximating slow roll with a patch of de Sitter where $\xi \simeq$ const yields

$$
A_{-\lambda}(\tau, \vec{k})=\frac{e^{\pi \xi / 2}}{\sqrt{2 k}} W_{-i \xi, 1 / 2}(2 i k \tau),
$$

where $W_{k, m}(z)$ is the Whittaker function, and we have imposed the Bunch-Davies vacuum initial conditions. While this approximation is valid, the average energy density of this field, and its Chern-Simons term, can be estimated as, for $\xi \geq 3$,

$$
\begin{aligned}
\rho_{E B}=\left\langle\frac{E^{2}+B^{2}}{2}\right\rangle & \simeq 1.3 \times 10^{-4} H^{4} \frac{e^{2 \pi \xi}}{\xi^{3}}, \\
\langle\vec{E} \cdot \vec{B}\rangle & \simeq-2.4 \times 10^{-4} \lambda H^{4} \frac{e^{2 \pi \xi}}{\xi^{4}} .
\end{aligned}
$$

We plot the evolution of $\rho_{E B}$ toward the end of the first inflationary stage, in Fig. 3.

The instability is driven by the slow roll-the tachyon is an instability of the transient background, not of the fundamental theory - and so the total energy deposited in the gauge field cannot exceed the inflaton kinetic energy. In particular, as inflation ends and $\phi_{1}$ settles into its minimum, the approximations (7) and (8) will cease to apply, with (7) reducing to the standard harmonic oscillator, as we noted above. We will take the transition from one limit to another to occur close to $\epsilon=-\dot{H} / H^{2} \sim 1$, or in other words, near the end of inflation, when $\dot{\phi}_{1}$ is almost maximal. This sounds counterintuitive, but from there on $\phi_{1}$ is decaying to its minimum, passing through zero quickly, and completely invalidating the approximations of (7), (8). At this point we will stop the numerical integration. ${ }^{2}$ Second, while the approximations (7) and (8) are valid, the $\mathrm{U}(1)$ vector gauge field energy density will backreact on the background evolution and the system will reach some equilibrium $[39,40]$. This does not significantly modify early inflation.

Towards the end of the first stage of inflation, as the field $\phi_{1}$ starts to move faster, the larger $\dot{\phi}_{1}$ results in a dramatic amplification of $\rho_{E B}$ cranking up the field strengths by as much as $\sim 10^{4}-10^{8}$, depending on the value of $f_{\phi}$. However the approximations break down at the exit of inflation, when $\rho_{E B} \sim \dot{\phi}_{1}^{2} / 2$ by energy conservation, and instead the growth in the plots turns around, "plateauing" near the end of inflation [38]. While we do not zoom in on the specifics of this behavior here, the "plateau" at the end of inflation in Fig. 3 is interspersed with characteristic features since as the field $\phi_{1}$ starts to decay and oscillate, the tachyonic instability of the $U(1)$ sector rapidly changes back and forth $[38,41] .{ }^{3}$ Those details could help tag the signals, warranting a more precise analysis.

The backreaction of the Chern-Simons term on the inflaton evolution may also alter and amplify scalar perturbations. In general, [40,43-45] note that this could lead to an enhanced primordial black hole production after inflation. Yet in the simplest setup which we rely on, with a single dark $\mathrm{U}(1)$ at scales $\sim 10-100 M_{\mathrm{GUT}}$, and without ultralight fields charged under U(1), it turns out that the enhancement of perturbations due to vector production is limited to about $\delta \rho / \rho \lesssim$ few $\times 10^{-2}$ near the end of the first stage of inflation, and miniscule earlier. This will limit distortions and $\mathrm{PBH}$ production rates. However, in extended models or with smaller $f_{\phi}$ those processes could be further enhanced.

\footnotetext{
${ }^{2}$ We expect that a more precise description of this transition can be pursued à la $\mathrm{WKB}$, by writing the formal solution to the last of Eqs. (6) as a contour integral and taking the limits $\xi \gg 1$ and $\xi \rightarrow 0$ to match (7) to the harmonic oscillator amplitude after inflation.

${ }^{3}$ See also [42] for earlier related results. Again, in Fig. 3 those effects are smoothed out.
} 


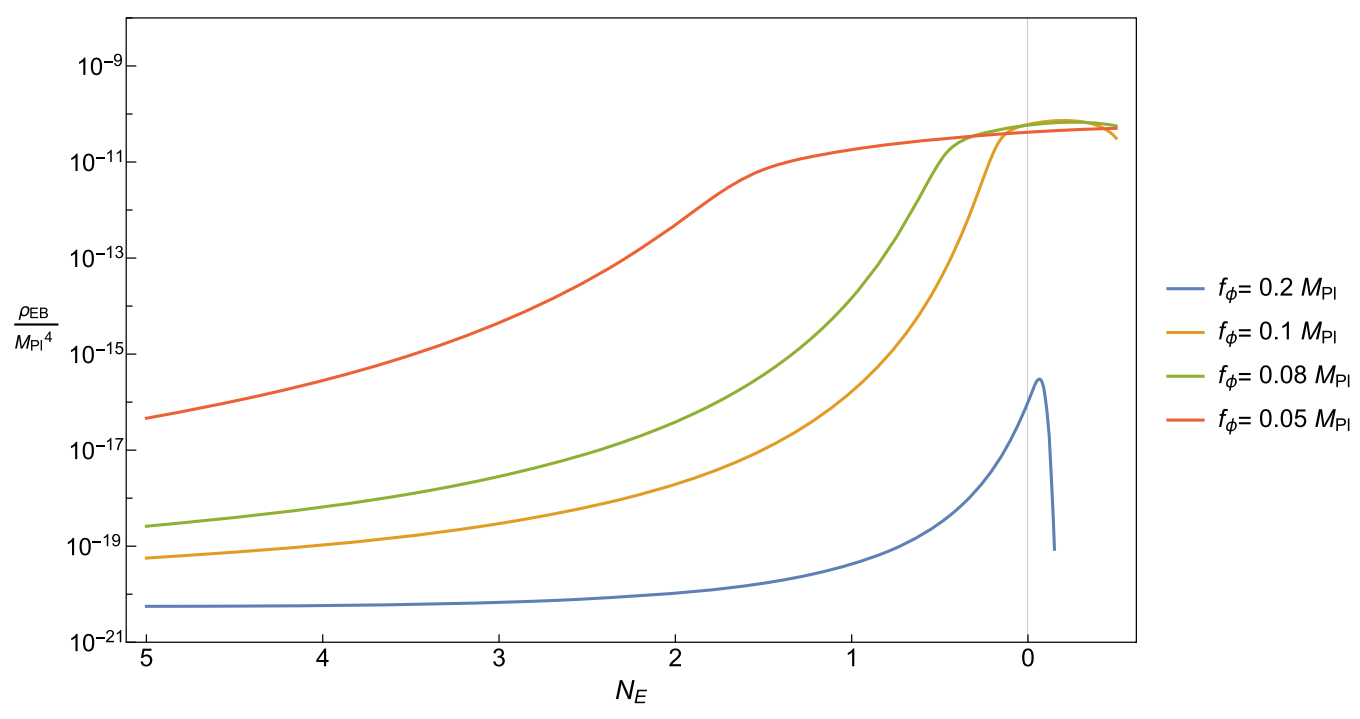

FIG. 3. Evolution at the end of the first inflationary stage of the energy density in gauge fields, Eq. (8). We denote by $N_{e}$ the number of $e$-folds before the end of the first stage of inflation, and we normalize the energy density to $M_{\mathrm{Pl}}^{4}$. The parameters of the potential are fixed for convenience to $M_{1}^{4}=2 \times 10^{-9} M_{\mathrm{Pl}}^{4}, \mu_{1}=M_{\mathrm{Pl}}, p=2 / 5$; at the CMB scales they lead to similar results as $\mu_{1}=0.1 M_{\mathrm{Pl}}$, which fit $\mathrm{CMB}$ perfectly. The vertical line denotes the end of inflation, and the solutions we show are not completely reliable there.

Here our main interest are the stochastic gravity waves produced near the end of the first stage of inflation. These modes are chiral, and their abundance can be estimated by [43]

$\Omega_{G W} \equiv \frac{\Omega_{r, 0}}{24} \Delta_{T}^{2} \simeq \frac{\Omega_{r, 0}}{12}\left(\frac{H}{\pi M_{\mathrm{Pl}}}\right)^{2}\left(1+4.3 \times 10^{-7} \frac{H^{2}}{M_{\mathrm{Pl}}^{2} \xi^{6}} e^{4 \pi \xi}\right)$,

where $\Omega_{r, 0}=8.6 \times 10^{-5}$ is the radiation abundance today, and the terms are evaluated at horizon crossing. The term in parenthesis adds to the standard tensors produced by metric fluctuations in de Sitter space the "secondary" production by the $U(1)$ vector gauge field. Its contribution tops the metric fluctuations near the end of the first stage of inflation.

Note that some of the modes produced just before the end of stage 1 would reenter the horizon during the intermediate matter-dominated stage. As a result, they would dilute by expansion during that epoch. Thus the formula for the abundance (9) should include an extra suppression factor. To estimate it, note that for subhorizon modes, the amplitude dilutes as $1 / \lambda \sim 1 / a$, where $a$ is the scale factor. The power in the modes is given by the square of the product of the frequency and the amplitude, since

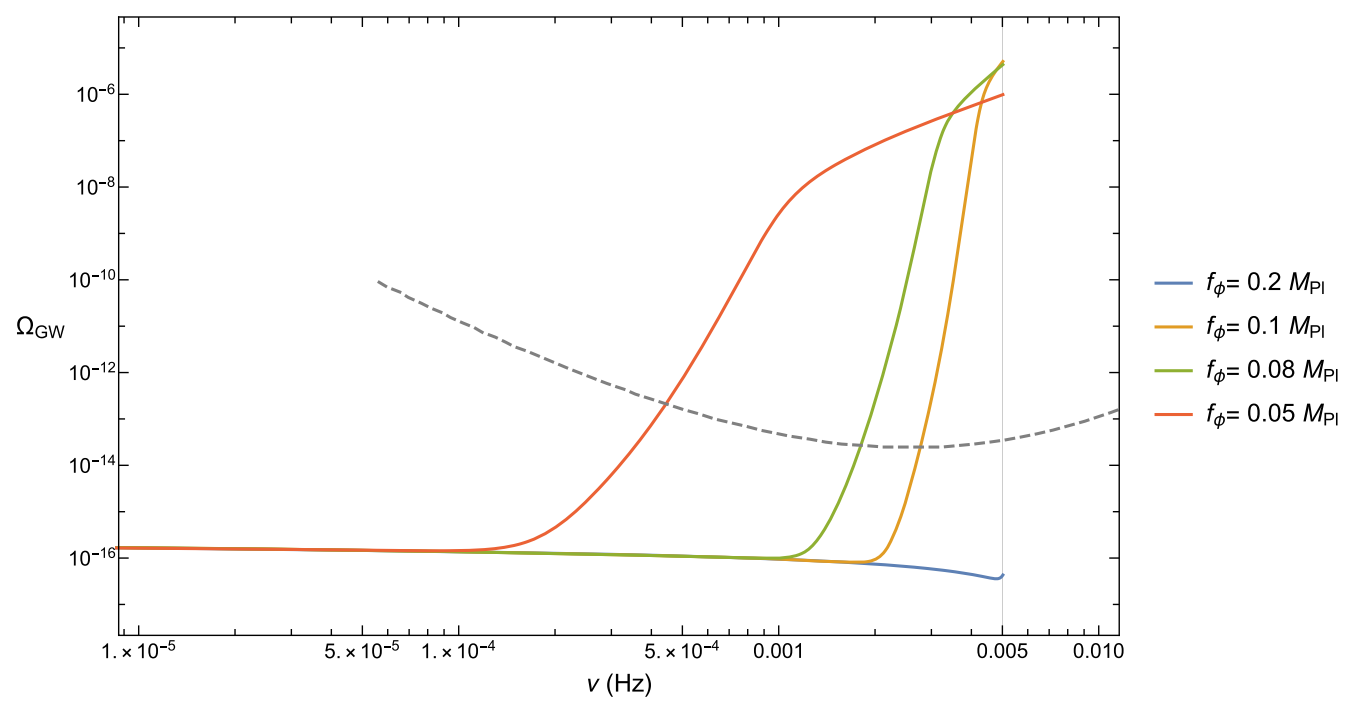

FIG. 4. Abundance of gravitational waves as a function of frequency, setting $N_{\mathrm{CMB}}=35$. The dashed grey line is the sensitivity of LISA, while the horizontal thin line is the bound from the number of relativistic degrees of freedom coming from BBN and CMB. The parameters of the potential are chosen as in Fig. 3. 
these modes behave as harmonic oscillators. Hence the suppression factor will be at most $\left(a_{1} / a_{2}\right)^{4}$ where $a_{1}$ is the scale factor at the end of stage 1 of inflation, and $a_{2}$ the scale factor at the mode refreezing after the beginning of the stage 2 of inflation. This factor is maximized for the shortest wavelength mode at the end of stage 1 , for which $k / a_{1} \sim H_{1}$. Since the mode freeze-out gives $k / a_{2} \simeq H_{2}$, this yields $a_{1} / a_{2} \lesssim H_{2} / H_{1}$. For a short intermediate stage this would suppress the power by at most a factor of 100 to 1000. This suppression would only be a subleading effect, which we ignored here. A more precise calculation of these effects would be in order, however, since this extra suppression actually helps to evade any conflicts with the BBN bound on gravity waves depicted in Fig. 4.

To get an idea how large these modes are, and at which scales they occur, we express $\Omega_{G W}$ as a function of the frequency observed in instruments at the present time. Since the comoving frequency is $\nu=k /(2 \pi)$, we obtain

$N=N_{\mathrm{CMB}}+\ln \frac{k_{\mathrm{CMB}}}{0.002 \mathrm{Mpc}^{-1}}-44.9-\ln \frac{\nu}{10^{2} \mathrm{~Hz}}$,

where $k_{\mathrm{CMB}}=0.002 \mathrm{Mpc}^{-1}$ is the $\mathrm{CMB}$ pivot scale, and $N_{\mathrm{CMB}}$ is the number of $e$-folds before the end of the first stage of inflation where the CMB scales froze out. We then replot the results using the new independent variable $\nu$ in Fig. 4. Beyond the end of inflation, the approximations breakdown; the curves would bend down there. Nevertheless it is clear that these modes are out there for LISA to see. Combined with the bounds on $r$ at the CMB scales, which are within reach of the future $\mathrm{CMB}$ polarization instruments, this makes our double-coaster extremely predictive and easy to falsify — or perhaps, confirm. Interestingly, not only would this be a search for a specific inflationary model, but also a quest for traces of naturalness on the sky.

\section{SUMMARY}

To summarize, we have described a very predictive theory of axion monodromy inflation. The main difference from the usual realizations of monodromy inflation is that inflation is not smooth, but happens in bursts as in rollercoaster cosmology. The example we focused on involves two axions which feature a little hierarchy between their masses, with initial conditions similar to Nflation.

Due to a little mass hierarchy, however, the axions fall out of slow roll at different times, leading to two stages of monodromy inflation separated by a stage of matter domination, during which the first inflaton oscillates briefly before the second inflaton takes over. Clearly these masses need to be tuned, although the required tuning does not need to be exceedingly precise. If the field ranges are $\mathcal{O}\left(M_{\mathrm{Pl}}\right)$, and axion potentials are flattened due to being close to the cutoff and in strong coupling regime, it is straightforward to arrange for the two stages of inflation to last $\mathcal{N} \sim 30-40$ and $\mathcal{N} \sim 20-30 e$-folds, respectively.

This yields the scalar and tensor perturbations at the largest scales which fit the CMB perfectly, with $0.02 \lesssim r \lesssim 0.06$, in the range of LiteBIRD and CMB-S4 experiments. In addition when the first inflaton couples to a hidden sector $U(1)$, which is quite generic in flux monodromy models where axions arise from dimensional reduction of higher rank $p$-forms, there will be an enhanced production of vectors near the end of the first stage of inflation. These modes source tensors during the short epoch of matter domination. These tensors are chiral, with wavelengths at the present time in the range of $10^{8} \mathrm{~km}$, and with amplitudes enhanced over the long wavelength modes by vector sources. They are a very loud signal for LISA. Hence we find that double monodromy inflation easily yields simultaneous signals accessible to future gravity wave instruments at different scales.

\section{ACKNOWLEDGMENTS}

We would like to thank A. Lawrence, E. Silverstein, L. Sorbo, and especially V. Domcke for useful discussions. N.K. is supported in part by the DOE Grant No. DESC0009999. A. W. is supported by the ERC Consolidator Grant STRINGFLATION under the HORIZON 2020 Grant Agreement No. 647995.
[1] A. H. Guth, The inflationary universe: A possible solution to the horizon and flatness problems, Adv. Ser. Astrophys. Cosmol. 3, 139 (1987).

[2] A. D. Linde, A new inflationary universe scenario: A possible solution of the horizon, flatness, homogeneity, isotropy and primordial monopole problems, Adv. Ser. Astrophys. Cosmol. 3, 149 (1987).

[3] A. Albrecht and P. J. Steinhardt, Cosmology for grand unified theories with radiatively induced symmetry breaking, Adv. Ser. Astrophys. Cosmol. 3, 158 (1987).
[4] C. Collins and S. Hawking, Why is the Universe isotropic?, Astrophys. J. 180, 317 (1973).

[5] N. Kaloper, A. Lawrence, and L. Sorbo, Anignoble approach to large field inflation, J. Cosmol. Astropart. Phys. 03 (2011) 023.

[6] E. Silverstein and A. Westphal, Monodromy in the CMB: Gravity waves and string inflation, Phys. Rev. D 78, 106003 (2008).

[7] L. McAllister, E. Silverstein, and A. Westphal, Gravity waves and linear inflation from axion monodromy, Phys. Rev. D 82, 046003 (2010). 
[8] N. Kaloper and L. Sorbo, A Natural Framework for Chaotic Inflation, Phys. Rev. Lett. 102, 121301 (2009).

[9] X. Dong, B. Horn, E. Silverstein, and A. Westphal, Simple exercises to flatten your potential, Phys. Rev. D 84, 026011 (2011).

[10] N. Kaloper and A. Lawrence, Natural chaotic inflation and ultraviolet sensitivity, Phys. Rev. D 90, 023506 (2014).

[11] L. McAllister, E. Silverstein, A. Westphal, and T. Wrase, The powers of monodromy, J. High Energy Phys. 09 (2014) 123.

[12] G. D'Amico, N. Kaloper, and A. Lawrence, Monodromy Inflation in the Strong Coupling Regime of the Effective Field Theory, Phys. Rev. Lett. 121, 091301 (2018).

[13] G. D'Amico and N. Kaloper, Rollercoaster cosmology, arXiv:2011.09489.

[14] M. Cicoli, S. Downes, B. Dutta, F. G. Pedro, and A. Westphal, Just enough inflation: Power spectrum modifications at large scales, J. Cosmol. Astropart. Phys. 12 (2014) 030 .

[15] M. Braglia, D. K. Hazra, F. Finelli, G. F. Smoot, L. Sriramkumar, and A. A. Starobinsky, Generating PBHs and small-scale GWs in two-field models of inflation, J. Cosmol. Astropart. Phys. 08 (2020) 001.

[16] G. Tasinato, An analytic approach to non-slow-roll inflation, arXiv:2012.02518.

[17] J. Fumagalli, S. Renaux-Petel, and L. T. Witkowski, Oscillations in the stochastic gravitational wave background from sharp features and particle production during inflation, arXiv:2012.02761.

[18] L. Anguelova, On primordial black holes from rapid turns in two-field models, arXiv:2012.03705.

[19] M. Braglia, X. Chen, and D. K. Hazra, Probing primordial features with the stochastic gravitational wave background, J. Cosmol. Astropart. Phys. 03 (2021) 005.

[20] C. Gordon, D. Wands, B. A. Bassett, and R. Maartens, Adiabatic and entropy perturbations from inflation, Phys. Rev. D 63, 023506 (2000).

[21] Y. M. Welling, Spectroscopy of two-field inflation, Ph.D. thesis, Leiden University, 2018.

[22] B. A. Campbell, N. Kaloper, R. Madden, and K. A. Olive, Physical properties of four-dimensional superstring gravity black hole solutions, Nucl. Phys. B399, 137 (1993).

[23] J. L. Cook and L. Sorbo, Particle production during inflation and gravitational waves detectable by ground-based interferometers, Phys. Rev. D 85, 023534 (2012); Erratum, Phys. Rev. D 86, 069901 (2012).

[24] L. Senatore, E. Silverstein, and M. Zaldarriaga, New sources of gravitational waves during inflation, J. Cosmol. Astropart. Phys. 08 (2014) 016.

[25] D. Polarski and A. A. Starobinsky, Spectra of perturbations produced by double inflation with an intermediate matter dominated stage, Nucl. Phys. B385, 623 (1992).

[26] S. Pi, M. Sasaki, and Y.-l. Zhang, Primordial tensor perturbation in double inflationary scenario with a break, J. Cosmol. Astropart. Phys. 06 (2019) 049.
[27] T. Banks, M. Dine, P. J. Fox, and E. Gorbatov, On the possibility of large axion decay constants, J. Cosmol. Astropart. Phys. 06 (2003) 001.

[28] P. Svrcek and E. Witten, Axions in string theory, J. High Energy Phys. 06 (2006) 051.

[29] M. Dias, J. Frazer, and A. Westphal, Inflation as an information bottleneck: A strategy for identifying universality classes and making robust predictions, J. High Energy Phys. 05 (2019) 065.

[30] S. Dimopoulos, S. Kachru, J. McGreevy, and J. G. Wacker, N-flation, J. Cosmol. Astropart. Phys. 08 (2008) 003.

[31] K. N. Abazajian et al. (CMB-S4 Collaborations), CMB-S4 science book, first edition, arXiv:1610.02743.

[32] P. Ade et al. (BICEP2, Keck Array Collaborations), BICEP2/ Keck Array x: Constraints on Primordial Gravitational Waves using Planck, WMAP, and New BICEP2/ Keck Observations through the 2015 Season, Phys. Rev. Lett. 121, 221301 (2018).

[33] N. Arkani-Hamed, L. Motl, A. Nicolis, and C. Vafa, The string landscape, black holes and gravity as the weakest force, J. High Energy Phys. 06 (2007) 060.

[34] H. Ooguri and C. Vafa, On the geometry of the string landscape and the swampland, Nucl. Phys. B766, 21 (2007).

[35] A. Hebecker, F. Rompineve, and A. Westphal, Axion monodromy and the weak gravity conjecture, J. High Energy Phys. 04 (2016) 157.

[36] N. Kaloper and L. Sorbo, Where in the string landscape is quintessence?, Phys. Rev. D 79, 043528 (2009).

[37] E. Witten, String theory dynamics in various dimensions, Nucl. Phys. B443, 85 (1995).

[38] V. Domcke, V. Guidetti, Y. Welling, and A. Westphal, Resonant backreaction in axion inflation, J. Cosmol. Astropart. Phys. 09 (2020) 009.

[39] M. M. Anber and L. Sorbo, Naturally inflating on steep potentials through electromagnetic dissipation, Phys. Rev. D 81, 043534 (2010).

[40] A. Linde, S. Mooij, and E. Pajer, Gauge field production in supergravity inflation: Local non-Gaussianity and primordial black holes, Phys. Rev. D 87, 103506 (2013).

[41] G. Dall'Agata, S. González-Martín, A. Papageorgiou, and M. Peloso, Warm dark energy, J. Cosmol. Astropart. Phys. 08 (2020) 032.

[42] S.-L. Cheng, W. Lee, and K.-W. Ng, Numerical study of pseudoscalar inflation with an axion-gauge field coupling, Phys. Rev. D 93, 063510 (2016).

[43] V. Domcke, F. Muia, M. Pieroni, and L. T. Witkowski, PBH dark matter from axion inflation, J. Cosmol. Astropart. Phys. 07 (2017) 048.

[44] J. Garcia-Bellido, M. Peloso, and C. Unal, Gravitational waves at interferometer scales and primordial black holes in axion inflation, J. Cosmol. Astropart. Phys. 12 (2016) 031.

[45] M. Peloso, L. Sorbo, and C. Unal, Rolling axions during inflation: Perturbativity and signatures, J. Cosmol. Astropart. Phys. 09 (2016) 001. 